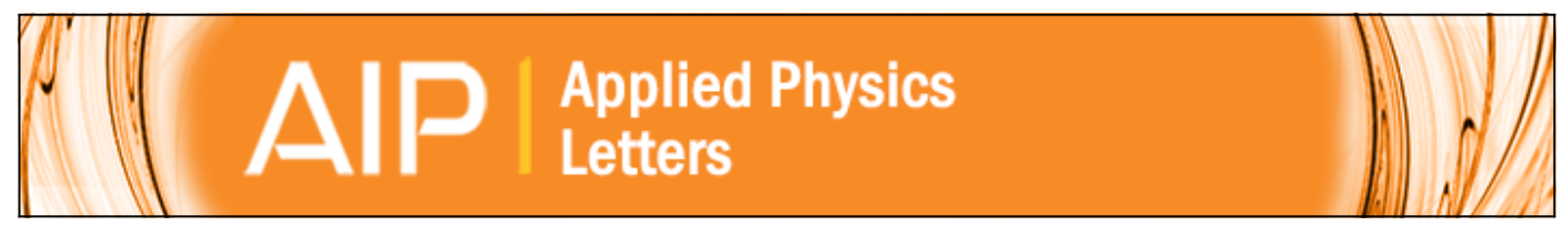

\title{
Ultrasound-modulated optical computed tomography of biological tissues
}

Jun Li and Lihong V. Wang

Citation: Applied Physics Letters 84, 1597 (2004); doi: 10.1063/1.1651330

View online: http://dx.doi.org/10.1063/1.1651330

View Table of Contents: http://scitation.aip.org/content/aip/journal/apl/84/9?ver=pdfcov

Published by the AIP Publishing

\section{Articles you may be interested in}

Lock-in camera based heterodyne holography for ultrasound-modulated optical tomography inside dynamic scattering media

Appl. Phys. Lett. 108, 231106 (2016); 10.1063/1.4953630

Region-of-interest diffuse optical tomography system

Rev. Sci. Instrum. 87, 013701 (2016); 10.1063/1.4939054

Cone beam x-ray luminescence computed tomography: A feasibility study

Med. Phys. 40, 031111 (2013); 10.1118/1.4790694

Three-dimensional optoacoustic tomography using a conventional ultrasound linear detector array: Whole-body tomographic system for small animals

Med. Phys. 40, 013302 (2013); 10.1118/1.4770292

Low-cost diffuse optical tomography for the classroom

Am. J. Phys. 80, 876 (2012); 10.1119/1.4739924

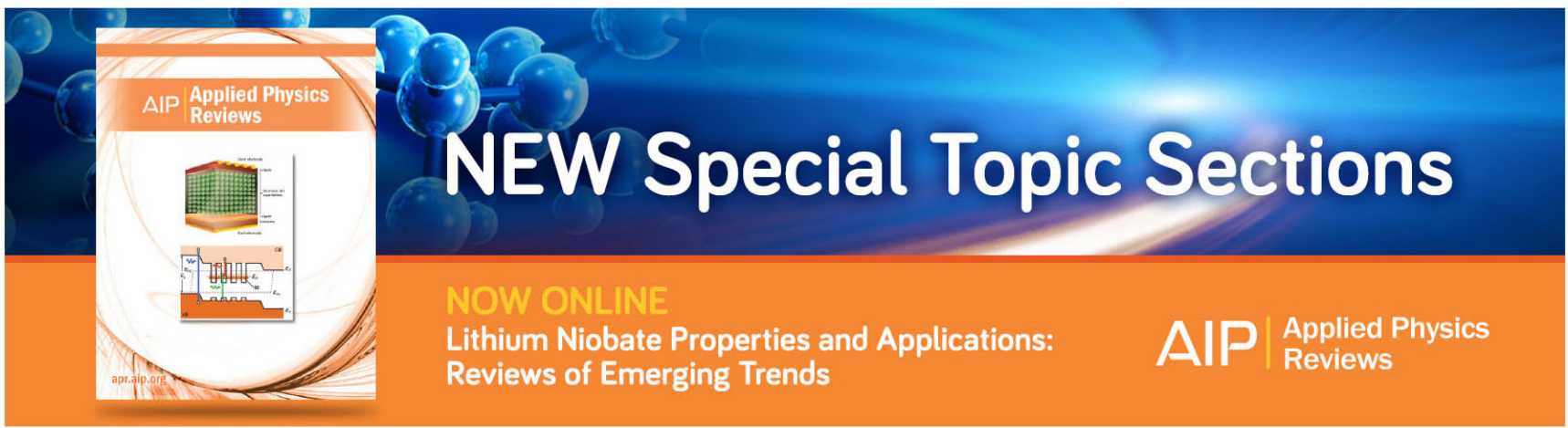




\title{
Ultrasound-modulated optical computed tomography of biological tissues
}

\author{
Jun Li and Lihong V. Wang ${ }^{\mathrm{a})}$ \\ Optical Imaging Laboratory, Department of Biomedical Engineering, Texas A\&M University, \\ College Station, Texas 77843-3120
}

(Received 12 August 2003; accepted 31 December 2003)

\begin{abstract}
An optical imaging technique called ultrasound-modulated optical computed tomography is demonstrated for tomographic imaging of biological tissues. Ultrasound-modulated optical signals are extracted from scattered light to provide projection data for the image reconstruction. A filtered back-projection algorithm is implemented to reconstruct an image reflecting optical tissue properties from angular and linear scans of an ultrasonic beam across a sample. This reconstruction-based imaging technique provides a way to obtain images of cross sections containing the scanned ultrasonic axis in biological tissues, which enables three-dimensional ultrasound-modulated optical imaging. The technique combines the contrast advantage of optical waves and the resolution advantage of ultrasonic waves. (C) 2004 American Institute of Physics. [DOI: 10.1063/1.1651330]
\end{abstract}

Taking advantage of optical contrast and ultrasonic resolution, ultrasound-modulated optical tomography ${ }^{1-5}$ is an attractive technique in the biomedical optical imaging field. In this technique, an ultrasonic beam is focused into biological tissue to modulate the coherent light crossing over the ultrasonic beam in the tissue. Because ultrasound is scattered much less than light in biological tissue, it can provide good spatial information for imaging in the optical diffusion regime. By detecting the ultrasound-modulated light, the optical properties related to the ultrasound location inside the tissue can be derived. Poor optical resolution, resulting from strong optical scattering of biological tissues, is avoided. By scanning a sample or an ultrasonic beam, one- (1D) or twodimensional (2D) images of a cross section perpendicular to the ultrasonic axis can be obtained directly. To obtain an image of a cross section containing the ultrasonic axis, Leveque-Fort ${ }^{6}$ reported scanning an ultrasonic beam axially; however, the resolution was poor because the axial dimension of the ultrasonic focal zone was quite long. Wang et al. ${ }^{7}$ and Yao et al. ${ }^{8}$ developed a technique called frequencyswept ultrasound-modulated optical tomography and achieved controllable spatial resolution along the ultrasonic axis, in which however a tradeoff exists between the resolution and the signal intensity. ${ }^{8}$ Current studies in the field are focusing on developing imaging techniques ${ }^{9}$ for obtaining the cross-section images as well as three-dimensional images. So far, no reconstruction algorithms have been employed in ultrasound-modulated optical tomography.

In this letter, we report a reconstruction-based imaging technique, ultrasound-modulated optical computed tomography (UMOCT), for cross-sectional imaging, with which three-dimensional images can be obtained. The technique was developed with inspiration from x-ray computed tomography $(\mathrm{CT})$. In $\mathrm{x}$-ray $\mathrm{CT}$, through multiple linear and angular scans around a sample carried out with an $\mathrm{x}$-ray sourcedetector pair, a cross-sectional image of the sample is reconstructed from the detected $\mathrm{x}$-ray intensities, which reflect the

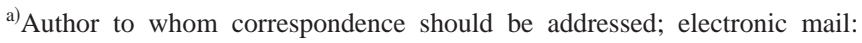
lwang@tamu.edu
}

attenuation properties along the x-ray paths in the sample. In ultrasound-modulated optical tomography, ultrasoundmodulated optical signals are generated in the volume that is occupied by the ultrasonic beam in the sample. Intensities of the ultrasound-modulated optical signals reflect the optical properties (optical absorption and scattering) in this volume. Motivated by the analogy with x-ray CT, we developed the UMOCT in which an ultrasonic beam was scanned linearly and angularly around a sample and a filtered back-projection algorithm was applied to reconstruct images of cross sections formed by the scanned ultrasonic axis. It should be noted that, in UMOCT, the scanning beam was an ultrasonic beam, and the signals to be detected were optical signals.

Figure 1(a) shows a schematic of the experimental setup. A laboratory coordinate system is shown to describe the sample in its original orientation. The optical axis was along the $y$ axis, and the ultrasonic axis was along the $z$ axis. A laser beam of $690 \mathrm{~nm}$ in wavelength and $11 \mathrm{~mW}$ in power from a diode laser (Melles Griot, 56IMS667) was expanded to $20 \mathrm{~mm}$ in diameter to illuminate the sample perpendicularly. The resulting power density was much lower than the safety limit $\left(\sim 200 \mathrm{~mW} / \mathrm{cm}^{2}\right) .{ }^{10}$ The coherence length of the laser was $\sim 6 \mathrm{~cm}$ when it was modulated at $1 \mathrm{MHz}$. Ultrasonic waves, generated by a focused ultrasonic transducer (UltranLab, VHP100-1-R38) with a $38 \mathrm{~mm}$ focal length in water and a $1-\mathrm{MHz}$ central response frequency, were coupled into the tissue sample through water in which

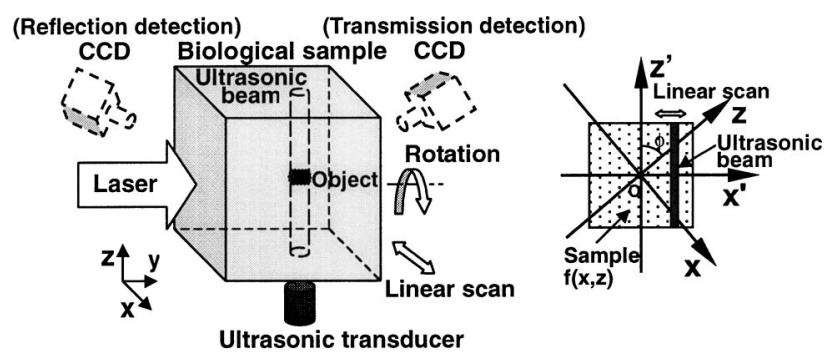

(a)

(b)

FIG. 1. Schematics of (a) the experimental configuration and (b) a cross section of a sample with coordinates considered in the reconstruction. 
the sample was partially immersed. The focal zone of the ultrasonic waves was $2 \mathrm{~mm}$ in diameter and $20 \mathrm{~mm}$ in length, in which the peak pressure was $\sim 10^{5} \mathrm{~Pa}$. Speckle patterns generated by the scattered light from the tissue were detected by a 12-bit digital charge-coupled device (CCD) camera (Dalsa, CA-D1-0256T) with $256 \times 256$ pixels. Then, ultrasound-modulated optical signals were extracted from the speckle patterns by the use of parallel speckle detection. ${ }^{5}$

An experiment with a transmission-detection configuration, in which the CCD camera and the incident laser beam were on opposite sides of the sample, as well an experiment with a reflection-detection configuration, in which the CCD camera and the incident laser beam were on the same side of the sample, were carried out. The UMOCT required relative linear and angular scans between the sample and the ultrasound. In the experiment, the position of the ultrasonic transducer was fixed. A sample holder was made to allow the sample to be rotated around the $y$ axis by a rotational stage and to be linearly scanned along the $x$ axis by a stepper motor. Figure 1(b) shows a cross section of the sample on the $x z$ plane, which has been rotated around the $y$ axis by an angle $\phi$ from its original orientation. A new coordinate system $\left(x^{\prime} y^{\prime} z^{\prime}\right)$ is set with the $z^{\prime}$ axis parallel to the ultrasonic beam. In the figure, the ultrasonic beam is located at a distance $x^{\prime}$ from the origin.

The detected ultrasound-modulated optical signal can be expressed as an integration of the signal coming from each segment along the $z^{\prime}$ axis $\left(z^{\prime}-\delta z^{\prime} / 2, z^{\prime}+\delta z^{\prime} / 2\right)$ in the ultrasonic column:

$$
s\left(\phi, x^{\prime}\right)=\int s_{\phi, x^{\prime}}\left(z^{\prime}\right) d z^{\prime},
$$

which is a Radon transform. The integrand can be expressed as $s_{\phi, x^{\prime}}\left(z^{\prime}\right)=C_{1} Q_{\phi, x^{\prime}}\left(z^{\prime}\right) m_{\phi, x^{\prime}}\left(z^{\prime}\right) G_{\phi, x^{\prime}}\left(z^{\prime}\right)$, where $C_{1}$ is a constant; $Q_{\phi, x^{\prime}}\left(z^{\prime}\right)$ represents the photon density in the segment centered at $z^{\prime}$, determined by the photon transport from the laser source to this segment; $m_{\phi, x^{\prime}}\left(z^{\prime}\right)$ is the depth of ultrasonic modulation related to the optical properties at $z^{\prime}$ in the ultrasonic column; and $G_{\phi, x^{\prime}}\left(z^{\prime}\right)$ represents the Green's function describing the transport of the ultrasoundmodulated photons from this segment to the detector. In the diffusion regime, $Q_{\phi, x^{\prime}}\left(z^{\prime}\right)$ and $G_{\phi, x^{\prime}}\left(z^{\prime}\right)$ have a weak dependence on $z^{\prime}$.

The signals detected at multiple projection angles are used as the projection data for the reconstruction of an image reflecting the optical properties of the sample, where a filtered back-projection algorithm is applied. The reconstructed image of the sample is expressed as

$$
\hat{f}(x, z)=\int_{0}^{\pi} \int_{-\infty}^{\infty} S(\phi, \omega)|\omega| \exp \left(i \omega x^{\prime}\right) d \omega d \phi,
$$

where $\omega$ is the spatial frequency in the $x^{\prime}$ direction and $S(\phi, \omega)$ is the Fourier transform of the signal $s\left(\phi, x^{\prime}\right)$. A Ram-Lak filter $|\omega|$ is used in the processing. The reconstructed values represent the relative intensities of the ultrasound-modulated optical signals. The lower intensities reflect higher attenuation in the sample and vice versa.

In the experiment, the step size of the linear scan along the $x^{\prime}$ axis was $1.2 \mathrm{~mm}$, and the increment of the angular scan was $5^{\circ}$. The signal-to-noise ratios in the measurement

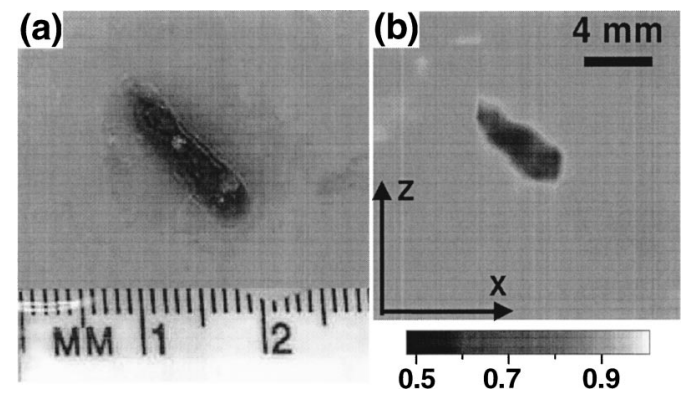

FIG. 2. Experimental result in the transmission-detection configuration: (a) Photograph of a cross section on the $x z$ plane located in the middle of a 14-mm-thick chicken-breast-tissue sample, in which an object made from blood vessel was buried; (b) reconstructed 2D image.

were $\sim 2$. To avoid the influence of acoustic heterogeneity on the ultrasound-modulated optical signal, we used allbiological-tissue samples: optically absorbent objects buried in background biological tissue were biological tissues as well. Both the objects and the background tissue had similar acoustic impedance. We first examined the technique in the transmission-detection configuration. To demonstrate that the technique depends on diffuse light, we placed the CCD camera at an angle of $25^{\circ}$ with respect to the optical axis to eliminate any contribution from ballistic photons. Figure 2(a) shows a photograph of a cross section of a chicken-breasttissue sample on the $x z$ plane. A piece of chicken blood vessel, which had a size of $\sim 8 \times 3 \mathrm{~mm}^{2}$ on the $x z$ plane and $\sim 2 \mathrm{~mm}$ in the $y$ direction, was buried in the middle of a 14-mm-thick sample. Figure 2(b) shows the reconstructed image, in which the buried object is clearly seen. The size and the orientation of the object in the image are in good agreement with the actual ones.

The technique was also examined in the reflectiondetection configuration. A chicken-breast-tissue sample was used, in which an object made from turkey muscle was buried $\sim 9 \mathrm{~mm}$ deep in the $y$ direction. The size of the object on the $x z$ plane was $\sim 4 \times 3 \mathrm{~mm}^{2}$. Figure 3 (a) shows a photograph of the imaged cross section of the sample, and Fig. 3(b) shows the reconstructed image. The object is clearly visible in the image and its size matches the actual size quite well. The reconstructed image was low-pass filtered. We evaluate the image resolution roughly with the width of the 10\%-90\% signal-intensity transition at the edge of the object. The width is $\sim 2 \mathrm{~mm}$, which indicates that the resolution in UMOCT is determined by the diameter of the ultrasonic
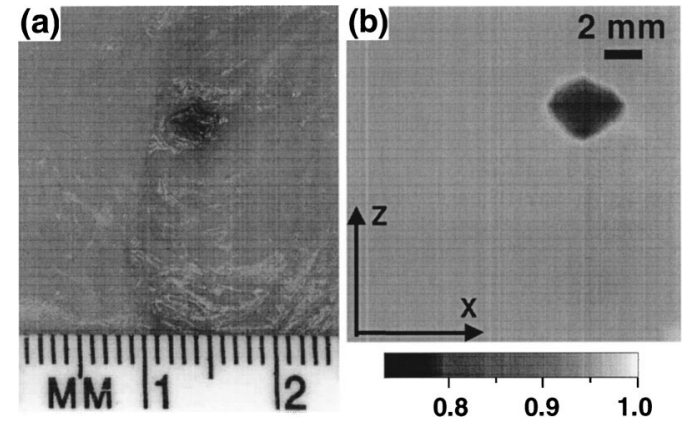

FIG. 3. Experimental result in the reflection-detection configuration: (a) Photograph of a cross section on the $x z$ plane located $\sim 9 \mathrm{~mm}$ deep in a chicken-breast-tissue sample, in which an object made from turkey muscle was buried; (b) reconstructed 2D image. 
focal zone $(2 \mathrm{~mm})$. As demonstrated in conventional ultrasound-modulated optical tomography, the resolution in the $y$ dimension is determined by the diameter of the ultrasonic focal zone as well. A better resolution can be obtained through the use of an ultrasonic transducer with a focal zone of smaller diameter.

The imaging of the cross section in the reflectiondetection configuration demonstrates the progress in ultrasound-modulated optical tomography. The reflection detection configuration is desired for medical imaging because of its convenient configuration. The results imply that the technique using the reflection detection configuration has good potential for application in medical imaging. In applications such as brain imaging, ${ }^{3}$ the incident laser beam and the detector will be located on the top of the head of a human being or an animal, and the ultrasonic transducer will be scanned around the head. It is expected that two-dimensional optical cross-sectional images as well as three-dimensional images at various depths in the brain, which have a controllable image resolution determined by the ultrasonic beam, can be obtained noninvasively with this technique. A future study will focus on the application of this technique to smallanimal brain imaging.

Compared with frequency-swept ultrasound-modulated tomography, this technique is a generic technique, which will work with any detection scheme in ultrasound-modulated optical tomography (e.g., single-detector detection, ${ }^{2}$ parallel speckle detection, ${ }^{1,5}$ or speckle-contrast detection $\left.{ }^{11,12}\right)$. In frequency-swept ultrasound-modulated optical tomography, an ultrasonic transducer with a broad bandwidth is required. Such a transducer may not be efficient for ultrasonic modulation because of the tradeoff between the efficiency and bandwidth of the transducer. In comparison, the resolution in UMOCT is determined by the diameter of the ultrasonic focal zone. A fine resolution can be achieved with sufficient signal intensities using a narrow-band high-efficiency ultrasonic transducer.

The method of X-ray CT has been applied in the optical regime for several years. ${ }^{13,14}$ However, our hybrid optical imaging technique is quite different in physics from those pure optical imaging techniques. In those applications, the scanning beam is a laser beam and the projection data are the intensity of the transmitted ballistic light, which cannot penetrate relatively thick biological tissues. By contrast, in our technique, the scanning beam is an ultrasonic beam and the projection data are the intensity of the diffuse light that is modulated by the ultrasonic beam. Although the scanning beam is an ultrasonic beam, the imaging is optically based because the contrast is based primarily on optical properties. Our technique extends the application of the method of x-ray $\mathrm{CT}$ in the area of optical imaging from the ballistic regime to the diffusion regime, with the aid of ultrasonic modulation.

In summary, we developed a technique called ultrasound-modulated optical computed tomography, which provides a way to obtain optical images of biological tissues in cross sections containing the scanned ultrasonic axis. The technique, which combines the advantages of optical contrast and ultrasonic resolution, can be implemented with any standard signal-detection scheme for ultrasonic modulation of coherent light in scattering media and can be applied directly to achieve three-dimensional images of biological tissues.

This project was sponsored in part by National Science Foundation Grant No. BES-9734491; Texas Higher Education Coordinating Board Grant No. 000512-0063-2001; and National Institutes of Health Grant No. R01 EB000712.

${ }^{1}$ S. Leveque, A. C. Boccara, M. Lebec, and H. Saint-Jalmes, Opt. Lett. 24, 181 (1999).

${ }^{2}$ A. Lev and B. G. Sfez, Opt. Lett. 27, 473 (2002).

${ }^{3}$ A. Lev, Z. Kotler, B. Sfez, J. Soustiel, and M. Feinsod, Acta Neurochir. Suppl. (Wien) 81, 295 (2002).

${ }^{4}$ L.-H. V. Wang, Phys. Rev. Lett. 87, 043903 (2001).

${ }^{5}$ J. Li and L.-H. V. Wang, Appl. Opt. 41, 2079 (2002).

${ }^{6}$ S. Leveque-Fort, Appl. Opt. 40, 1029 (2000).

${ }^{7}$ L.-H. Wang and G. Ku, Opt. Lett. 23, 975 (1998).

${ }^{8}$ G. Yao, S. Jiao, and L.-H. Wang, Opt. Lett. 25, 734 (2000).

${ }^{9}$ B. C. Forget, F. Ramaz, M. Atlan, J. Selb, and A. C. Boccara, Appl. Opt. 42, 1379 (2003).

${ }^{10}$ American National Standards Institute, American National Standard for the Safe Use of Lasers, Standard Z136.1-1993 (ANSI, New York, 1993).

${ }^{11}$ J. Li, G. Ku, and L.-H. V. Wang, Appl. Opt. 41, 6030 (2002).

${ }^{12}$ J. Li, S. Sakadžić, G. Ku, and L.-H. V. Wang, Appl. Opt. 42, 4088 (2003).

${ }^{13}$ B. Devaraj, M. Takeda, M. Kobayashi, M. Usa, K. P. Chan, Y. Watanabe, T. Yuasa, T. Akatsuka, M. Yamada, and H. Inaba, Appl. Phys. Lett. 69, 3671 (1996).

${ }^{14}$ R. G. Kelly, K. J. Jordan, and J. J. Battista, Med. Phys. 25, 1741 (1998). 